\title{
Intraarticular Botulinum Toxin A for Refractory Painful Total Knee Arthroplasty: A Randomized Controlled Trial
}

\author{
Jasvinder A. Singh, Maren L. Mahowald, and Siamak Noorbaloochi \\ Rheumatology Section, Medicine Service, and Division of Rheumatology, and Center for Chronic \\ Disease Outcomes Research, Department of Medicine, Veterans Affairs Medical Center, \\ University of Minnesota, Minneapolis, Minnesota; Departments of Health Sciences Research and \\ Orthopedics, Mayo Clinic School of Medicine, Rochester, Minnesota; Birmingham Veterans \\ Affairs Medical Center and University of Alabama at Birmingham, Birmingham, Alabama, USA.
}

\section{Abstract}

Objective-To assess short-term efficacy of single intraarticular botulinum toxin (IA-BoNT/A) injection in patients with chronically painful total knee arthroplasty (TKA) in a randomized, placebo-controlled, triple-blind study.

Methods-Patients with chronic TKA pain (pain > 6 on 0-10 scale and > 6 months post-TKA) evaluated in and referred from orthopedic surgery clinics were recruited. The primary outcome, proportion of patients with clinically meaningful decrease of at least 2 points on $0-10$ visual analog scale (VAS) for pain, was compared between treatment groups at 2 months using comparison of proportions test and for all efficacy timepoints (2, 3, and 4 months) using generalized estimating equations (GEE). Secondary outcomes of global assessment, function, and quality of life were compared using GEE, duration of pain relief by t-test, and adverse events by chi-square test.

Results-In total, 54 patients with 60 painful TKA were randomized, with main analyses restricted to one TKA per patient (49 TKA in 49 patients). Mean age was 67 years, $84 \%$ were men, and mean duration of TKA pain was 4.5 years. A significantly greater proportion of patients (71\%) in the IA-BoNT/Agroup compared to IA-placebo (35\%) achieved clinically meaningful reduction in VAS pain at 2 months $(\mathrm{p}=0.028)$ and at all efficacy timepoints $(\mathrm{p}=0.019)$. Duration of meaningful pain relief was significantly greater after IA-BoNT/A, 39.6 days (SD 50.4) compared to IA-placebo, 15.7 days (SD 22.6; $\mathrm{p}=0.045$ ). Statistically significantly better scores were seen in IA-BoNT/A vs IA-place-bo for all efficacy timepoints for the following outcomes: "very much improved" on physician global assessment of change $(\mathrm{p}=0.003)$; Western Ontario McMaster Osteoarthritis Index physical function $(p=0.026)$, stiffness $(p=0.004)$, and total scores $(\mathrm{p}=0.024)$; and Short-Form 36 pain subscale score $(\mathrm{p}=0.049)$. Number of total and serious adverse events was similar between groups, with no patients in either group with new objective motor or sensory deficits during followup.

Copyright $\odot 2010$. All rights reserved.

Address correspondence to Dr. J.A. Singh, University of Alabama, Faculty Office Tower 805B, 510 - 20th Street S., Birmingham, AL 35294. Jasvinder.md@ gmail.com.

J.A. Singh, MBBS, MPH, Rheumatology Section, Medicine Service, Veterans Affairs Medical Center, and Division of Rheumatology, Department of Medicine, University of Minnesota; Departments of Health Sciences Research and Orthopedics, Mayo Clinic School of Medicine; Birmingham Veterans Affairs Medical Center and University of Alabama at Birmingham; M.L. Mahowald, MD,

Rheumatology Section, Medicine Service, Veterans Affairs Medical Center, and Division of Rheumatology, Department of Medicine, University of Minnesota; S. Noorbaloochi, PhD, Center for Chronic Disease Outcomes Research, Veterans Affairs Medical Center and Department of Medicine, University of Minnesota. 
Conclusion-In this single-center randomized trial, single IA-BoNT/A injection provided clinically meaningful short-term improvements in pain, global assessment, and function in patients with chronic painful TKA. A multicenter trial is needed to confirm these findings.

\section{Key Indexing Terms \\ TOTAL KNEE ARTHROPLASTY; INTRAARTICULAR; BOTULINUM TOXIN A; RANDOMIZED CONTROLLED TRIAL; PAIN; FUNCTION}

Total knee arthroplasty (TKA) improves pain, function, and health-related quality of life (HRQOL) in patients with end-stage knee arthritis1. TKA has an annual volume of 500,000/ year in the United States, with a 6-fold projected increase to 3.48 million/year by 20302 . However, $8 \%-13 \%$ of patients $(40,000-65,000$ patients/yr) report persistent moderate to severe pain in the prosthetic knee after TKA3,4, which may be due to infection, loosening or instability, or an unknown cause5. Revision surgery may relieve pain secondary to infection, loosening, or instability. Limited medical or surgical treatment options are currently available to patients with painful TKA from an unknown cause.

Substance P (SP) and calcitonin gene-related protein (CGRP) are neuropeptides that may play a role in post-TKA knee pain. Nerve fibers with positive immunostaining to SP, CGRP, and Neurokinin A were found in bone prosthesis interface membranes obtained during revision surgery for painful primary hip arthroplasty ${ }^{6}$. Joint fluid SP levels were elevated in painful knee joints with osteoarthritis (OA) that underwent TKA, but not in normal/ asymptomatic contralateral knees7. Significantly greater pain relief after knee arthroplasty was seen in patients with an elevated preoperative joint fluid SP level compared to patients with normal levels7.

Botulinum toxin A (BoNT/A) injections have anticholinergic effect (responsible for muscleparalyzing action) and an independent antinociceptive effect8. This dual action was noted in patients with cervical dystonia9 and headaches10.An antinociceptive effect of local or intramuscular injections of BoNT/A has been reported in randomized controlled trials (RCT) of patients with chronic tennis elbow11, myofascial pain12,13, post-stroke shoulder pain14, postherpetic neuropathic pain15, diabetic neuropathic pain16, painful bladder syndrome17, and painful external hemorrhoids ${ }^{18}$. Additionally, intraarticular (IA) injections of BoNT/A (IA-BoNT/A) were shown to have an antinociceptive effect in uncontrolled studies for patients with chronic refractory knee, shoulder, and ankle joint pain ${ }^{19}, 20,21$, and in RCT in patients with refractory pain due to shoulder arthritis 22 or knee arthritis ${ }^{23}$. In a systematic review of RCT of shoulder pain, we found that botulinum toxin injection was superior to placebo for short-term pain relief at 3-6 months ${ }^{24}$.

Laboratory studies demonstrate that BoNT/A can modulate neurotransmitter release and neurogenic inflammation. This may explain its independent antinociceptive effect.

Neurogenic inflammation is a phenomenon of antidromic stimulation of primary afferent fiber that induces neuropeptide release in the periphery associated with vasodilation, protein extravasation, and stimulation of inflammatory cells. SP and CGRP are the main mediators of neurogenic inflammation ${ }^{25}$. In vitro studies showed that BoNT/A inhibited stimulated CGRP release from rat trigeminal ganglia ${ }^{26}$ and capsaicin-stimulated SP release from embryonic rat dorsal root ganglia neurons ${ }^{27}$. BoNT/A inhibited stimulated SP release ${ }^{28}$ and CGRP release ${ }^{28,29}$ in models of acute and chronic inflammation of isolated rat urinary bladders, BoNT/A injections into the rat paw reduced formalin-induced paw edema, tissue glutamate release, and spinal cord electrical excitations ${ }^{30}$. 
In this randomized, placebo-controlled study, we investigated the short-term efficacy of a single IA-BoNT/A injection for reduction in pain severity in patients with chronically painful TKA with no evidence of prosthesis infection or fracture.

\section{MATERIALS AND METHODS}

\section{Study design and schedule}

This study's primary purpose was to determine the short-term antinociceptive efficacy of IA-BoNT/A at 2 months, as measured by a visual analog scale (VAS). The selection of 2 months as the primary endpoint was based on our observation of significant pain relief at 2 months postinjection in our open-label study of IA-BoNT/A for painful TKA ${ }^{31}$. Other efficacy endpoints included 2-week and 1-month telephone interviews (especially to assess the onset of pain relief) and 2-, 3-, and 4-month in-clinic assessments. The 6-month evaluation was primarily to calculate the duration of pain relief and for safety monitoring. Inclusion and exclusion criteria are listed in Table 1. All patients underwent complete examination to rule out infection and other surgical correctable causes of pain in painful TKA (fracture, malalignment, loosening, etc.) by the referring orthopedic surgeon.

\section{Randomization, blinding, and injection procedure}

The research pharmacist prepared computerized randomization with permuted blocks of 4 patients each. Joints were randomized in 1:1 ratio to experimental treatment with IA injection of 100 units BoNT/A diluted in $5 \mathrm{ml}$ sterile normal saline (BoNT/A group) or placebo treatment with IA injection of $5 \mathrm{ml}$ sterile normal saline and IA injection.

The research pharmacist prepared the treatment and placebo syringes using a strict standardized protocol. Freeze-dried botulinum toxin A (Botox, Allergan Inc., Irvine, CA, USA) was reconstituted immediately prior to injection, in $5 \mathrm{ml}$ preservative-free sterile $0.9 \%$ normal saline (100 units $/ 5 \mathrm{ml})$ without agitation. Placebo and botulinum toxin injections were transparent and could not be differentiated. Patients, investigators (PI, blinded investigators performing assessments, research associates), and the statistician were blinded in this triple-blind study.

The PI injected the affected TKA joint using the standardized medial or lateral approach32, demonstrated as highly accurate ${ }^{33}$. IA medication delivery was verified by joint fluid aspiration in all patients, which was sent for gram stain and culture in all cases, when enough specimen was obtained (> 90\% of aspirates, which were all negative for both tests).

\section{Outcomes}

All outcomes were chosen a priori and were listed in the protocol on clinicaltrials.gov (NCT00403273). The primary efficacy outcome was the proportion of responders at 2 months. Responder status was defined as clinically meaningful pain relief of 2-point reduction in $0-10$ VAS pain score ${ }^{34}$. Pain severity was assessed on a $0-10 \mathrm{~cm}$ VAS $(0=$ no pain, $10=$ worst possible pain), a valid, reliable measure of pain that is sensitive to change $35,36,37$.

The secondary outcome measures included physicians' global assessment of change, rated on a validated 7 -point verbal descriptor nominal scale $(1=$ very much improved, $2=$ much improved, $3=$ minimally improved, $4=$ no change, $5=$ minimally worse, $6=$ much worse, 7 $=$ very much worse $)^{38}$.

Onset and duration of pain relief were defined as time from the injection to onset and duration of patient-reported clinically meaningful pain relief, defined as a 20-point decrease 
on Western Ontario McMaster Osteoarthritis Index (WOMAC) pain scale39. WOMAC pain scale has been validated for telephone administration40; it was administered at all timepoints, including 2-week and 4-week telephone interviews (when VAS pain could not be determined).

Function was measured by the physical function subscale of a patient-reported, lower extremity-specific validated measure, the WOMAC ${ }^{41}$. WOMAC consists of 5 pain items, 2 stiffness items, and 17 physical function items, each graded on a Likert scale (none, mild, moderate, severe, extreme). Raw scores from the pain, stiffness, and physical function subscales are added to obtain a total WOMAC score. Each subscale and total WOMAC score is transformed on 0-100 scale, higher score being worse.

Objective function assessment was done using 2 validated objective tests and by measuring active knee flexion and extension. Timed-stands test (TST) was the time to perform sit to stand 10 times without using arms to push up. Mean value ranged from 17 to 21 seconds in 60- to 80-year-old subjects42. Timed-up-and-go (TUG) test was the time for a patient to get up from an armchair, walk a distance of 3 meters, turn, walk to the chair, and sit. Independently mobile patients perform the test in $<20$ seconds 43 . Active knee flexion (lower $=$ better) and extension (neutral, 180 degrees; higher $=$ better) were measured using the universal goniometer (Conzett model; PhysioERP, Laval, Quebec, Canada) with the patient in the supine position 44 .

The Medical Outcomes Study Short-Form 36 (SF-36; range 0-100, higher score = better HRQOL), a valid and reliable generic HRQOL measure, has been validated in musculoskeletal conditions ${ }^{45}$. It has 8 subscale scores for physical HRQOL, including bodily pain and emotional/mental health HRQOL46.

We used the Short-form McGill Pain Questionnaire (SF-MPQ), a validated qualitative multidimensional measure of pain ${ }^{47}$. It measures sensory pain (11 items, score 0-33) and affective dimension of pain (4 items, score $0-12$ ), summed to obtain a total pain score (score range $0-45$; higher score $=$ worse pain $)$.

Changes in analgesic medications from baseline to 2 months were calculated for 3 most common medications/groups including acetaminophen, nonsteroidal antiinflammatory drugs (NSAID), and opioids. The WHO Defined Daily Dose (DDD) was used to convert doses of various NSAID to an equivalent analgesic DDD ${ }^{48}$. For example, $1.2 \mathrm{~g}$ ibuprofen $=0.1 \mathrm{~g}$ indomethacin $=0.5 \mathrm{~g}$ naproxen $=1 \mathrm{DDD}$. Opioid doses were converted to morphine equivalents using the standard conversion factors 49.

\section{Safety outcomes}

We assessed safety during study followup visits by interviewing patients regarding occurrence of any adverse events, including a standardized checklist of common side effects of BoNT/A. Patients underwent a bilateral lower extremity neurosensory examination at each clinic visit by a blinded physician examiner. This included manual muscle strength testing, deep tendon reflexes (knee and ankle), and sensory neurological examination (light touch, pinprick, vibration, position sense, and hot and cold discrimination). Adverse events were rated for severity (mild/nonserious or serious). A serious adverse event was defined as fatal, life-threatening, permanently disabling, or requiring hospitalization.

\section{Statistical analyses}

Baseline characteristics were compared using t-tests and chi-square tests. For the analysis of primary outcome, VAS pain at 2 months, we compared the proportion of responders (those with 2-point reduction in VAS pain score) in the 2 groups using comparison of proportions. 
VAS pain was also analyzed at all efficacy timepoints using generalized estimating equation (GEE) modeling. We used GEE for between-group comparisons in continuous and categorical secondary outcomes at all efficacy endpoints, adjusted for baseline scores.

The main analyses were done for the 49 patients with 49 painful TKA, since this provided us with required sample size and did not violate the assumption of independence (which may be violated by including 2 joints in a patient). We performed sensitivity analyses by including patients with bilateral injections (included 5 patients with 10 simultaneous TKA and 1 with sequential second TKA injection; total 60 TKA) using GEE, adjusting for paired baseline measurements (right, left).

We used independent sample t-tests for between-group comparisons of changes in doses of acetaminophen, NSAID, and opioids at 2 months and of onset and duration of clinically meaningful pain relief. The chi-square tests compared the incidence of adverse events in the treatment and placebo groups. A p $<0.05$ was considered statistically significant.

\section{Sample size}

Nineteen patients per group were needed for $80 \%$ power and 24 patients per group for $90 \%$ power (assuming 25\% loss to followup), to detect a difference of $43 \%$ in proportion of patients reporting a clinically meaningful improvement in pain. This was based on previously published data that $43 \%$ patients taking placebo reported significant improvement in pain on a $0-100$ WOMAC pain scale ${ }^{50}$ vs $86 \%$ in IA-BoNT/A group in an open-label case series 31 .

The study was approved by the Minneapolis Medical Center Human Studies Committee. Both veterans and nonveterans were recruited from orthopedic clinics at Minneapolis Medical Center and community orthopedic clinics. The trial was listed on clinicaltrials.gov (NCT00403273).A US Food and Drug Administration Investigational New Drug (IND) mandate was obtained (BB-IND-11493) for IA injection as a new route of administration.

\section{RESULTS}

\section{Demographic and clinical characteristics}

Figure 1 shows the structure of the study, according to CONSORT (Consolidated Standards of Reporting Trials). Of the 60 TKA randomized, 5 patients had simultaneous bilateral TKA and 1 patient had sequential bilateral TKA injected. Outcomes are subject to within-patient dependence for bilateral TKA. Therefore, the main analyses are based on the 49 patients with 49 TKA (including the first TKA of the only patient with sequential enrollment of bilateral painful TKA). Of these 49 patients, 23 were randomized to IA-BoNT/A and 26 to IA-placebo. Sensitivity analyses included all 60 TKA.

The demographic and clinical characteristics are presented in Table 2. The mean duration of TKA pain was 4.5 years (SD 4.8, range $1 \pm 20 \mathrm{yrs}$ ). The 2 study groups were similar at baseline (including all outcome measures except WOMAC pain; Table 2).

\section{Primary outcome, responders: proportion with clinically meaningful reduction in VAS pain}

At 2 months, $71 \%$ of patients (95\% CI 49\%, 92\%) in the IA-BoNT/A group vs 35\% (95\% CI $15 \%, 54 \%$ ) in the IA-placebo group were responders, a statistically significant difference of $36 \%$ between treatment groups (95\% CI for difference, $7 \%, 65 \% ; \mathrm{p}=0.025$; Table 3 ). The proportion of responders at all efficacy timepoints up to 4 months was significantly greater in the IA-BoNT/A vs the IA-placebo group (using the GEE; $p=0.019$; Table 3). These results did not change with the sensitivity analyses that included bilateral TKA for 2- 
month primary endpoint (with $p=0.027$ ) and for all efficacy time-points $(p=0.037)$. Of the 12 responders in the botulinum toxin group with 2-point reduction in VAS pain at 2 months (17 patients had VAS assessments at both timepoints), 11 patients were still responders at 3 months and 8 patients at 4 months. In comparison, of the 8 placebo responders at 2 months (23 patients had VAS assements at both timepoints), 4 were still responders at 3 months and 5 at 4 months. The median (25th percentile, 75 th percentile) VAS pain scores in the IABoNT/A vs the IA-placebo group were as follows: preinjection, $7.0(6.3,8.3)$ vs $7.5(6.5$, $8.0)$; at 2 months, $4.5(3,6.5)$ vs $6.5(3.3,7.5)$; at 3 months, $4.0(2.5,6.5)$ vs $5.5(3.5,8.0)$; and at 4 months, $5.3(4,6.5)$ vs $5.5(3.3,6.8)$.

\section{Secondary outcomes}

Physician global assessment of change showed significantly more improvement in the IABoNT/A compared to the IA-placebo group at 2 months: $30 \%$ (95\% CI 10\%, 50\%) in the IA-BoNT/A group were "very much improved" vs $0 \%$ in the IA-placebo group (95\% CI not assessed; $p=0.005)$. Sensitivity analyses did not change the interpretation $(p=0.002)$. Including all efficacy timepoints, physician global assessment of change was significantly better in the IA-BoNT/A than in the placebo group $(\mathrm{p}=0.003)$.

The duration of meaningful pain relief was significantly greater in the IA-BoNT/A group at 39.6 (50.4) days vs 15.7 (22.6) days in IA-placebo $(\mathrm{p}=0.045)$. The onset of meaningful pain relief in the IA-BoNT/A group was 66.5 days (SD 47, median 56 days).

WOMAC physical function $(p=0.026)$, stiffness $(p=0.004)$, and total scores $(p=0.024)$ were significantly better in the IA-BoNT/A group compared to IA-placebo at all efficacy timepoints (Table 4). Timed-up-and-go (TUG) test showed a trend toward better scores in the IA-BoNT/A group vs IA-placebo $(\mathrm{p}=0.14)$. The timed-stands test (TST), active flexion, and extension were not significantly different between groups (Table 4).

The SF-36 pain subscale score was significantly better/higher in the IA-BoNT/A group compared to IA-placebo $(p=0.049$; Table 4$)$. No significant differences were noted in other SF-36 subscale scores. The McGill affective dimension score showed a marginal (nonsignificant) trend favoring IA-BoNT/A $(\mathrm{p}=0.08$; Table 4). McGill sensory and total scores showed no significant differences.

There were minimal changes in analgesic intake at the 2-month followup. NSAID dose at 2 months decreased by $0.1 \mathrm{DDD}$ (SD 0.32) in IA-BoNT/A vs an increase by $0.02 \mathrm{DDD}$ (SD $0.29)$ in IA-placebo, a trend toward significance $(\mathrm{p}=0.21)$. Respective changes in acetaminophen dose [60 $\mathrm{mg}$ decrease (1308) vs $186 \mathrm{mg}$ decrease (636), respectively; $\mathrm{p}=$ 0.71 ] and morphine equivalents [0.66 increase (15.4) vs 0.50 decrease (1.7); $p=0.67$ ] showed no significance.

\section{Safety outcomes}

The frequency of all adverse events was similar in the BoNT/Agroup compared to the placebo group $(\mathrm{p}=0.76$; Table 5). Local adverse events, including increased pain in the study joint, occurred in 6 IA-BoNT/A patients and 2 IA-placebo patients. Transient muscle weakness around study joint or knee "giving out" was reported by 4 and 2 patients, respectively. Objective examination revealed no evidence of new lower extremity motor or sensory deficits or signs of joint inflammation in any patient during followup. The most common systemic adverse events (dry mouth, upper respiratory symptoms, accidental injury, headache, chest pain, and back pain) were similar in both groups (Table 5). Most adverse effects were attributed to preexisting comorbid conditions and/or treatments and were unrelated to the joint injection. More patients in the IA-placebo than the IA-BoNT/A group had serious adverse events, although the difference was not statistically significant ( $p$ 
$=0.16$ ). There was 1 death in the IA-BoNT/A group, a 64-year-old woman with history of obesity, obstructive sleep apnea, depression, deep vein thrombosis, multiple pulmonary emboli, status-post right above-knee amputation for an infected arthroplasty after failed arthrodesis, on life-long anticoagulation. She was unable to ambulate and undergo rehabilitation for right knee prosthesis because of a painful left TKA. She was hospitalized with altered sensorium 1.5 months after the injection. She had an intracerebral bleed secondary to a fusiform vertebral artery aneurysm with dissection and a high prothrombin time. She died 1 day after admission. This was judged to be related to the cerebral aneurysm and anticoagulation and not related to the intraarticular injection.

\section{DISCUSSION}

This is the first study to demonstrate the short-term efficacy of a single intraarticular injection of botulinum toxin in patients with chronic painful TKA, when infection, loosening, wear, and other established causes of painful knee replacement have been definitively ruled out. We found that IA botulinum toxin injection led to a greater proportion of patients (71\%) with clinically meaningful reduction in pain severity 2 months after injection compared to IA placebo (35\%). Clinically meaningful reduction in pain severity lasted significantly longer in the IA-BoNT/A compared to the placebo group. However, this clinically meaningful pain relief lasted a mean of 39 days in the botulinum toxin group, implying that we may need a dose higher than 100 units and/or repeat injections to sustain meaningful pain relief for long enough duration. Mean VAS pain scores trended to, but did not achieve statistical significance between the groups, likely due to heterogeneity of pain relief and the small sample size. Longer duration of pain relief is very desirable for patients with chronic painful TKA. In addition, risk of introducing infection in TKA must be weighed against the benefits of pain relief. IA botulinum toxin injection led to significantly more improvements in physician global assessment and WOMAC physical function, stiffness, and total scores, compared to the placebo injection. Use of IA BoNT/A is not yet approved by the US Food and Drug Administration. We do not recommend clinical use of IA BoNT/A for painful TKA until more studies have been performed.

Our study provides a novel approach to short-term treatment of pain in patients with painful TKA, where infection and mechanical causes of pain have been ruled out. The presence of SP and CGRP nerve fibers in bone-prosthesis interface membrane in patients with painful arthroplasty ${ }^{6}$ and inhibition of SP and CGRP peripherally and centrally by BoNT/A in animal models of acute and chronic inflammation ${ }^{26,27,28,29,30}$ provides 1 potential explanation of antinociceptive action of BoNT/A injections in patients with painful TKA. Our study suggests that neuropeptides may be important mediators of pain in patients with painful TKA without documented infection, loosening, or other mechanical reasons. Studies to investigate the mechanism of action of IA-BoNT/A in painful TKA and the role of SP and other neuropeptides in painful TKA are under way.

Patient-reported function and stiffness were significantly better after IA-BoNT/A compared to IA-placebo. The finding that WOMAC subscales were sensitive to change after IA injection of a painful TKA adds to the literature of its sensitivity to change in patients with $\mathrm{OA}$ and knee arthroplasty 52,53 . The TUG test showed a trend toward significance favoring IA-BoNT/A, and the TST showed no difference between groups. The lack of significance in objective tests of function (TUG, TST) may be due to a small sample size, to almost normal baseline values leading to a floor effect (for TUG), or to lack of sensitivity to change with this therapy. Similarly, knee flexion and extension measures did not change much in either group, which indicates either lack of effect of treatment or lack of sensitivity to change. A longer followup may be required to identify significant objective functional improvement, as noted for other joints ${ }^{54,55}$. Similarly, the lack of differences in SF-36 subscale scores, 
except for the SF-36 pain score, may be due to several reasons, including the lack of the sensitivity of this QOL measure to change with an intraarticular therapy, the well reported fact that it is influenced by comorbidities other than TKA, and/or the need for longer followup. Lack of significant difference in WOMAC pain scores between treatment groups may be at least partially due to higher WOMAC pain scores in the placebo group at the baseline than in the botulinum group.

Our study provides short-term safety data for IA botulinum toxin injection. The frequency of all adverse effects and of serious adverse events was similar in the 2 treatment groups. Local side effects of transient increase in knee pain and knee weakness/giving out were slightly more frequent in the IA-BoNT/A group, but the difference did not reach statistical significance. One patient died secondary to dissection of vertebral artery aneurysm while receiving chronic anticoagulation therapy 1.5 months after IA-BoNT/A injection. The death was determined to be related to the underlying vascular disease and not the IA injection.

Our study has several strengths and limitations. The study was randomized and had robust estimates that did not change with sensitivity analyses. Multiple comparisons were avoided by performing only repeated measures analyses of a priori chosen outcomes and not performing any subgroup analyses (only 20 comparisons). The main study limitations are small sample size and short to intermediate followup. Results may not be generalizable to women, since most patients $(80 \%)$ were men. This is partly because more veterans than nonveterans were enrolled in our study; however, we recruited several nonveterans and a greater proportion of women than a typical Veterans Affairs study to increase the generalizability of these findings. The lack of significant difference in some secondary outcomes between treatment groups may be due to small sample size and/or to improvements noted in the placebo group. The placebo response may possibly be due to "Hawthorne effect" (observation of improvement in patients when under observation as part of clinical studies) or regression to the mean in this population with moderately severe pain at baseline. However, we are unsure of the exact reason for the continued improvement in pain in the placebo group up to 4 months. This is similar to previously reported placebo responses with IA injections for knee $\mathrm{OA}^{56,57 .}$

Our randomized controlled trial showed that a single injection of IA-BoNT/A was associated with a significant reduction in pain and improvement in patient-reported physical function and stiffness in patients with chronically painful TKA, where infection and mechanical causes of pain have been ruled out. No significant improvement in objective measures of knee function was noted. Treatment of painful TKA with no clear etiology is an important clinical problem with limited treatment options. During the short 6-month followup, the adverse effects were similar to placebo and were mostly mild. Our study results suggest that a larger multicenter study of IA-BoNT/A is needed to confirm these findings, to better understand its mechanism of action, and to examine whether repeated injections provide persistent pain relief in patients with painful TKA.

\section{Acknowledgments}

We thank Dr. Terence Gioe and Dr. Richard Schmidt from Orthopedic Surgery for evaluating and referring patients; Anita Ngo, Ruth Brady, Peter Majeski, and Patrick Fitzgerald for help in collecting data; Amy Anderson for proofreading the manuscript; Beverly Manderfield for designing the Access database; Dr. Sherine Gabriel and Dr. Patrick Mantyh for helpful comments; and the patients for their participation.

Supported by the NIH CTSA Award 1 KL2 RR024151-01 (Mayo Clinic Center for Clinical and Translational Research), Arthritis Foundation North Central Chapter Grant, University of Minnesota Academic Health Center Seed Grant, and Minnesota Medical Foundation Grant. Dr. Singh and Dr. Mahowald have received research and travel grants from Allergan Pharmaceuticals, Irvine, CA, for other research projects. Dr. Mahowald served as a consultant to Allergan Pharmaceuticals. 


\section{REFERENCES}

1. Ethgen O, Bruyere O, Richy F, Dardennes C, Reginster JY. Health-related quality of life in total hip and total knee arthroplasty. A qualitative and systematic review of the literature. J Bone Joint Surg Am 2004;86:963-974. [PubMed: 15118039]

2. Kurtz S, Ong K, Lau E, Mowat F, Halpern M. Projections of primary and revision hip and knee arthroplasty in the United States from 2005 to 2030. J Bone Joint Surg Am 2007;89:780-785. [PubMed: 17403800]

3. Brander VA, Stulberg SD, Adams AD, Harden RN, Bruehl S, Stanos SP, et al. Predicting total knee replacement pain: a prospective, observational study. Clin Orthop Relat Res 2003;416:27-36. [PubMed: 14646737]

4. Singh JA, Gabriel S, Lewallen D. The impact of gender, age, and preoperative pain severity on pain after TKA. Clin Orthop Relat Res 2008;466:2717-2723. [PubMed: 18679762]

5. Brown EC 3rd, Clarke HD, Scuderi GR. The painful total knee arthroplasty: diagnosis and management. Orthopedics 2006;29:129-136. quiz 37-8. [PubMed: 16485456]

6. Ahmed M, Bergstrom J, Lundblad H, Gillespie WJ, Kreicbergs A. Sensory nerves in the interface membrane of aseptic loose hip prostheses. J Bone Joint Surg Br 1998;80:151-155. [PubMed: 9460973]

7. Pritchett JW. Substance P level in synovial fluid may predict pain relief after knee replacement. J Bone Joint Surg Br 1997;79:114-116. [PubMed: 9020458]

8. Jabbari B. Botulinum neurotoxins in the treatment of refractory pain. Nat Clin Pract Neurol 2008;4:676-685. [PubMed: 19043424]

9. Jankovic J, Schwartz K. Botulinum toxin injections for cervical dystonia. Neurology 1990;40:277280. [PubMed: 2300249]

10. Gobel H, Heinze A, Heinze-Kuhn K, Jost W. Evidence based medicine: Botulinum toxin A in migraine and tension type headache. J Neurol 2001;248 Suppl:34-38. [PubMed: 11357239]

11. Keizer S, Rutten H, Pilot P, Morre H, Os J, Verburg A. Botulinum toxin injection versus surgical treatment for tennis elbow: a randomized pilot study. Clin Orthop 2002;401:125-131. [PubMed: 12151889]

12. Porta M. A comparative trial of botulinum toxin type A and methylprednisolone for the treatment of myofascial pain syndrome and pain from chronic muscle spasm. Pain 2000;85:101-105. [PubMed: 10692608]

13. Wheeler AH, Goolkasian P, Gretz SS. A randomized, double-blind, prospective pilot study of botulinum toxin injection for refractory, unilateral, cervicothoracic, paraspinal, myofascial pain syndrome. Spine 1998;23:1662-1666. discussion 7. [PubMed: 9704373]

14. Marco E, Duarte E, Vila J, Tejero M, Guillen A, Boza R, et al. Is botulinum toxin type A effective in the treatment of spastic shoulder pain in patients after stroke? A double-blind randomized clinical trial. J Rehabil Med 2007;39:440-447. [PubMed: 17624477]

15. Ranoux D, Attal N, Morain F, Bouhassira D. Botulinum toxin type A induces direct analgesic effects in chronic neuropathic pain. Ann Neurol 2008;64:274-283. [PubMed: 18546285]

16. Yuan RY, Sheu JJ, Yu JM, Chen WT, Tseng IJ, Chang HH, et al. Botulinum toxin for diabetic neuropathic pain: a randomized double-blind crossover trial. Neurology 2009;72:1473-1478. [PubMed: 19246421]

17. Kuo HC, Chancellor MB. Comparison of intravesical botulinum toxin type A injections plus hydrodistention with hydrodistention alone for the treatment of refractory interstitial cystitis/ painful bladder syndrome. BJU Int 2009;104:657-661. [PubMed: 19338543]

18. Patti R, Arcara M, Bonventre S, Sammartano S, Sparacello M, Vitello G, et al. Randomized clinical trial of botulinum toxin injection for pain relief in patients with thrombosed external haemorrhoids. Br J Surg 2008;95:1339-1343. [PubMed: 18844269]

19. Mahowald ML, Singh JA, Dykstra D. Long term effects of intra-articular botulinum toxin A for refractory joint pain. Neurotox Res 2006;9:179-188. [PubMed: 16785116]

20. Singh JA, Mahowald ML. Intra-articular botulinum toxin A as an adjunctive therapy for refractory joint pain in patients with rheumatoid arthritis receiving biologics: a report of two cases. Joint Bone Spine 2009;76:190-194. [PubMed: 18952480] 
21. Singh JA, Mahowald ML, Kushnaryov A, Goelz E, Dykstra D. Repeat injections of intra-articular botulinum toxin A for the treatment of chronic arthritis joint pain. J Clin Rheumatol 2009;15:3538. [PubMed: 19131763]

22. Singh JA, Mahowald ML, Noorbaloochi S. Intra-articular botulinum toxin A for refractory shoulder pain: a randomized, double-blinded, placebo-controlled trial. Transl Res 2009;153:205216. [PubMed: 19375681]

23. Boon AJ, Smith J, Dahm DL, Sorenson EJ, Larson DR, Fitz-Gibbon PD, et al. Efficacy of intraarticular botulinum toxin type A in painful knee osteoarthritis: a pilot study. Phys Med Rehabil 2010;2:268-276.

24. Singh JA, Fitzgerald P. Botulinum toxin for shoulder pain [in press]. Cochrane Database Syst Rev. 2010

25. Birklein F, Schmelz M. Neuropeptides, neurogenic inflammation and complex regional pain syndrome (CRPS). Neurosci Lett 2008;437:199-202. [PubMed: 18423863]

26. Meng J, Wang J, Lawrence G, Dolly JO. Synaptobrevin I mediates exocytosis of CGRP from sensory neurons and inhibition by botulinum toxins reflects their anti-nociceptive potential. J Cell Sci 2007;120:2864-2874. [PubMed: 17666428]

27. Purkiss J, Welch M, Doward S, Foster K. Capsaicin-stimulated release of substance P from cultured dorsal root ganglion neurons: involvement of two distinct mechanisms. Biochem Pharmacol 2000;59:1403-1406. [PubMed: 10751549]

28. Lucioni A, Bales GT, Lotan TL, McGehee DS, Cook SP, Rapp DE. Botulinum toxin type A inhibits sensory neuropeptide release in rat bladder models of acute injury and chronic inflammation. BJU Int 2008;101:366-370. [PubMed: 18184328]

29. Rapp DE, Turk KW, Bales GT, Cook SP. Botulinum toxin type a inhibits calcitonin gene-related peptide release from isolated rat bladder. J Urol 2006;175:1138-1142. [PubMed: 16469640]

30. Cui M, Khanijou S, Rubino J, Aoki KR. Subcutaneous administration of botulinum toxin A reduces formalin-induced pain. Pain 2004;107:125-133. [PubMed: 14715398]

31. Singh J, Mahowald M, Krug H, Gioe T, Santos E, Schmidt R. Intra-articular botulinum toxin A for painful total knee arthroplasty (TKA) [abstract]. Arthritis Rheum 2006;54 Suppl:S541-S542.

32. Courtney P, Doherty M. Joint aspiration and injection. Best Pract Res Clin Rheumatol 2005;19:345-369. [PubMed: 15939363]

33. Lopes RV, Furtado RN, Parmigiani L, Rosenfeld A, Fernandes AR, Natour J. Accuracy of intraarticular injections in peripheral joints performed blindly in patients with rheumatoid arthritis. Rheumatology 2008;47:1792-1794. [PubMed: 18820311]

34. Farrar JT, Portenoy RK, Berlin JA, Kinman JL, Strom BL. Defining the clinically important difference in pain outcome measures. Pain 2000;88:287-294. [PubMed: 11068116]

35. Gallagher EJ, Liebman M, Bijur PE. Prospective validation of clinically important changes in pain severity measured on a visual analog scale. Ann Emerg Med 2001;38:633-638. [PubMed: 11719741]

36. Turk DC, Rudy TE, Sorkin BA. Neglected topics in chronic pain treatment outcome studies: determination of success. Pain 1993;53:3-16. [PubMed: 8316386]

37. Bijur PE, Silver W, Gallagher EJ. Reliability of the visual analog scale for measurement of acute pain. Acad Emerg Med 2001;8:1153-1157. [PubMed: 11733293]

38. Farrar JT, Young JP Jr, LaMoreaux L, Werth JL, Poole RM. Clinical importance of changes in chronic pain intensity measured on an 11-point numerical pain rating scale. Pain 2001;94:149_ 158. [PubMed: 11690728]

39. Tubach F, Ravaud P, Baron G, Falissard B, Logeart I, Bellamy N, et al. Evaluation of clinically relevant changes in patient reported outcomes in knee and hip osteoarthritis: the minimal clinically important improvement. Ann Rheum Dis 2005;64:29-33. [PubMed: 15208174]

40. Bellamy N, Campbell J, Hill J, Band P. A comparative study of telephone versus onsite completion of the WOMAC 3.0 osteoarthritis index. J Rheumatol 2002;29:783-786. [PubMed: 11950022]

41. Bellamy N, Buchanan WW, Goldsmith CH, Campbell J, Stitt LW. Validation study of WOMAC: a health status instrument for measuring clinically important patient relevant outcomes to antirheumatic drug therapy in patients with osteoarthritis of the hip or knee. J J Rheumatol 1988;15:1833-1840. 
42. Newcomber K, Krug H, Mahowald M. Validity and reliability of the timed stands test for patients with rheumatoid arthritis and other chronic diseases. J Rheumatol 1993;20:21-27. [PubMed: 8441160]

43. Podsiadlo D, Richardson S. The timed "Up \& Go": a test of basic functional mobility for frail elderly persons. J Am Geriatr Soc 1991;39:142-148. [PubMed: 1991946]

44. Gagey OJ, Gagey N. The hyperabduction test. J Bone Joint Surg Br 2001;83:69-74. [PubMed: $11245541]$

45. Katz JN, Larson MG, Phillips CB, Fossel AH, Liang MH. Comparative measurement sensitivity of short and longer health status instruments. Med Care 1992;30:917-925. [PubMed: 1405797]

46. Ware JE Jr, Sherbourne CD. The MOS 36-item short-form health survey (SF-36). I. Conceptual framework and item selection. Med Care 1992;30:473-483. [PubMed: 1593914]

47. Melzack R. The short-form McGill Pain Questionnaire. Pain 1987;30:191-197. [PubMed: 3670870]

48. Frette C, Barrett-Connor E, Clausen JL. Effect of active and passive smoking on ventilatory function in elderly men and women. Am J Epidemiol 1996;143:757-765. [PubMed: 8610685]

49. Watanabe KI, Minabe Y, Ashby CR Jr, Katsumori H. Effect of acute administration of various 5HT receptor agonists on focal hippocampal seizures in freely moving rats. Eur J Pharmacol 1998;350:181-188. [PubMed: 9696406]

50. Bellamy N, Bell MJ, Goldsmith CH, Pericak D, Walker V, Raynauld JP, et al. The effectiveness of hylan G-F 20 in patients with knee osteoarthritis: an application of two sets of response criteria developed by the OARSI and one set developed by OMERACT-OARSI. Osteoarthritis Cartilage 2005;13:104-110. [PubMed: 15694571]

51. Sangha O, Stucki G, Liang MH, Fossel AH, Katz JN. The Self-Administered Comorbidity Questionnaire: a new method to assess comorbidity for clinical and health services research. Arthritis Rheum 2003;49:156-163. [PubMed: 12687505]

52. Wright JG, Young NL. A comparison of different indices of responsiveness. J Clin Epidemiol 1997;50:239-246. [PubMed: 9120522]

53. Gentelle-Bonnassies S, Le Claire P, Mezieres M, Ayral X, Dougados M. Comparison of the responsiveness of symptomatic outcome measures in knee osteoarthritis. Arthritis Care Res 2000;13:280-285. [PubMed: 14635296]

54. van der Windt DA, Koes BW, Deville W, Boeke AJ, de Jong BA, Bouter LM. Effectiveness of corticosteroid injections versus physiotherapy for treatment of painful stiff shoulder in primary care: randomised trial. BMJ 1998;317:1292-1296. [PubMed: 9804720]

55. Carette S, Moffet H, Tardif J, Bessette L, Morin F, Fremont P, et al. Intraarticular corticosteroids, supervised physiotherapy, or a combination of the two in the treatment of adhesive capsulitis of the shoulder: a placebo-controlled trial. Arthritis Rheum 2003;48:829-838. [PubMed: 12632439]

56. Lo GH, LaValley M, McAlindon T, Felson DT. Intra-articular hyaluronic acid in treatment of knee osteoarthritis: a meta-analysis. JAMA 2003;290:3115-3121. [PubMed: 14679274]

57. Espallargues M, Pons JM. Efficacy and safety of viscosupplementation with Hylan G-F 20 for the treatment of knee osteoarthritis: a systematic review. Int J Technol Assess Health Care 2003;19:41-56. [PubMed: 12701938] 


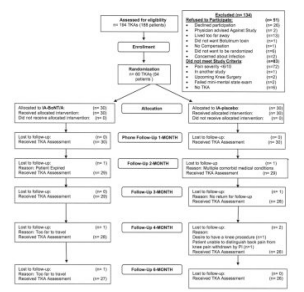

Figure 1.

The structure of the study. Five patients had simultaneous bilateral TKA injected, 1 patient had sequential TKA injection (i.e., enrolled his left and right TKA in the study at 2 different times). Baseline, 2, 3, 4, and 6-month followup visits were in-clinic or required hospitalization. 
Table 1

Study inclusion and exclusion criteria.

Inclusion criteria

1 Adults who underwent TKA > 6 months before study entry

2 Chronic painful TKA for $>3$ months with pain $\geq 6$ on a 10-point numerical pain rating scale

3 Failed treatments including oral pain medications and were not surgical candidates

4 Negative investigation for an infectious etiology by the referring orthopedic surgeon with one or more of the following - normal inflammatory markers, including normal erythrocyte sedimentation rate, or C-reactive protein; normal clinical examination; and/or culture-negative joint fluid aspirate

Exclusion criteria

1 History of myasthenia gravis, Eaton-Lambert syndrome, amyotrophic lateral sclerosis, or other known diseases of the neuromuscular junction or motor neuron disease

2 Concomitant use of aminoglycoside or agents that interfere with neuromuscular junction transmission

3 Women who were pregnant or breast-feeding

$4 \quad$ Known allergy or sensitivity to the study medication

5 Recent or ongoing alcohol or drug abuse

6 Known, uncontrolled systemic disease

7 Concurrent participation in other drug study 
Table 2

Baseline demographic and clinical characteristics of study participants.

\begin{tabular}{|c|c|c|}
\hline Characteristic & IA-BoNT/A, $n=23^{*}$, mean $(\mathrm{SD})$ or $\%$ & IA-placebo, $n=26^{*}$, mean $(\mathrm{SD})$ or $\%$ \\
\hline Mean age, (SD), yrs & $67.1(10.1)$ & $66.8(11.5)$ \\
\hline Men, n (\%) & $18 / 23(78)$ & $23 / 26(88)$ \\
\hline Caucasian, n (\%) & $22 / 23(96)$ & $25 / 26(96)$ \\
\hline Mean comorbidity index ${ }^{* *}$ & $13.0(5.5)$ & $12.5(28.7)$ \\
\hline Mean pain duration of total knee arthroplasty (TKA), yrs & $4.8(5.1)$ & $4.1(4.6)$ \\
\hline Primary/revision TKA & $16 / 7$ & $21 / 5$ \\
\hline \multicolumn{3}{|l|}{ Current treatment, $\mathrm{n}(\%)^{\dagger}$} \\
\hline Nonsteroidal antiinflammatory drugs & $15 / 23(65)$ & $21 / 26(81)$ \\
\hline Narcotics & $10 / 23(43)$ & $10 / 26(38)$ \\
\hline Acetaminophen & $11 / 23(48)$ & $8 / 26(31)$ \\
\hline Other ${ }^{\dagger \dagger}$ & $8 / 23(35)$ & $8 / 26(31)$ \\
\hline \multicolumn{3}{|l|}{ Primary outcome } \\
\hline VAS pain severity, $0-10$ & $7.2(1.1)$ & $7.5(1.3)$ \\
\hline \multicolumn{3}{|l|}{ Secondary outcomes } \\
\hline WOMAC physical function, $0-100$ & $56.5(8.9)$ & $63.3(17.5)$ \\
\hline WOMAC pain, $0-100$ & $58.0(13.6)$ & $67.1(15.3)$ \\
\hline WOMAC stiffness, 0-100 & $64.1(20.4)$ & $64.4(18.9)$ \\
\hline WOMAC total, $0-100$ & $63.2(9.3)$ & $70.6(16.8)$ \\
\hline Timed Stands Test, seconds & $49.9(31.5)$ & $50.8(29.3)$ \\
\hline Timed Up and Go Test, seconds & $18.3(13.8)$ & $18.5(12.7)$ \\
\hline Active flexion & $83.3(22.5)$ & $86.2(16.7)$ \\
\hline Active extension & $170.7(10.9)$ & $167.8(10.0)$ \\
\hline McGill affective dimension & $6.1(2.9)$ & $5.3(3.1)$ \\
\hline McGill sensory dimension & $16.7(6.5)$ & $17.3(6.8)$ \\
\hline McGill total score & $22.8(8.2)$ & $22.7(8.8)$ \\
\hline \multicolumn{3}{|c|}{ Data from 49 patients with 49 TKA, i.e., only one TKA per patient. } \\
\hline \multicolumn{3}{|c|}{ Comorbidity assessed using self-reported validaed comorbidity index 51 . } \\
\hline \multicolumn{3}{|l|}{${ }^{\dagger}$ Many patients were taking multiple medications. } \\
\hline
\end{tabular}

IA: intraarticular; BoNT/A: botulinum toxin A; VAS: visual analog scale; WOMAC: Western Ontario McMaster University Arthritis Index. 
Table 3

Percentage of responders, i.e., patients with 2-point decrease in visual analog scale pain severity at each efficacy timepoint.

\begin{tabular}{lccc}
\hline Group & 2-month Proportion (95\% & 3-month Proportion (95\% CI) & 4-month Proportion (95\% CI) \\
\hline IA-BoNT/A & $71(49,92)$ & $62(41,83)$ & $50(27,73)$ \\
IA-placebo & $35(15,54)$ & $43(22,64)$ & $55(33,77)$ \\
Difference (IA-BoNT/A-IA-placebo) & $36(7,65)$ & $19(-11,49)$ & $-5(-37,27)$ \\
\hline
\end{tabular}

$$
\text { * }
$$

The difference between botulinum toxin and placebo groups was statistically significant at 2 months $(\mathrm{p}=0.025)$. The difference between botulinum toxin and placebo groups was also statistically significant when all 3 timepoints $(2,3$ and 4 months) were included $(p=0.019)$, using generalized estimating equations analyses. 


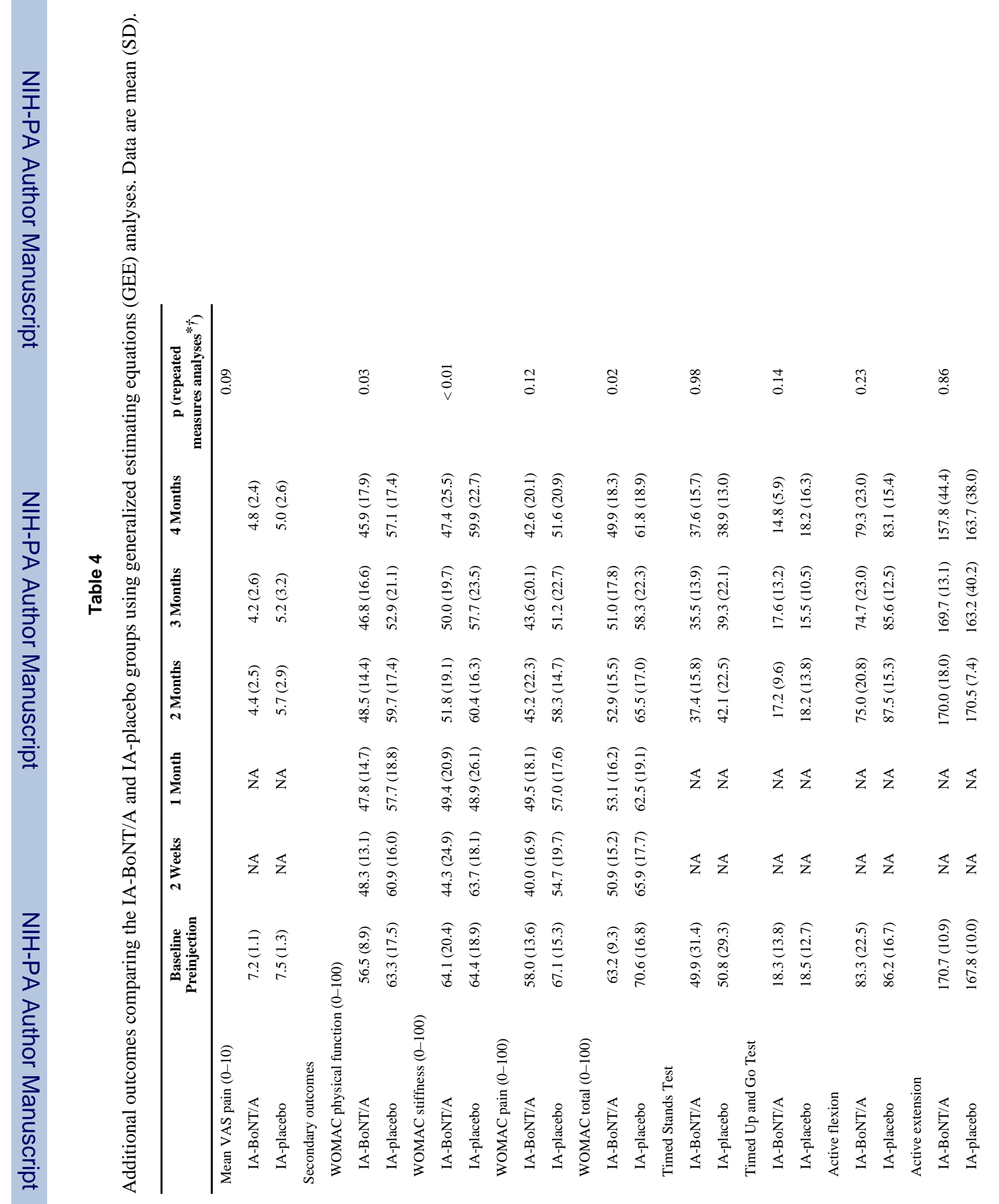




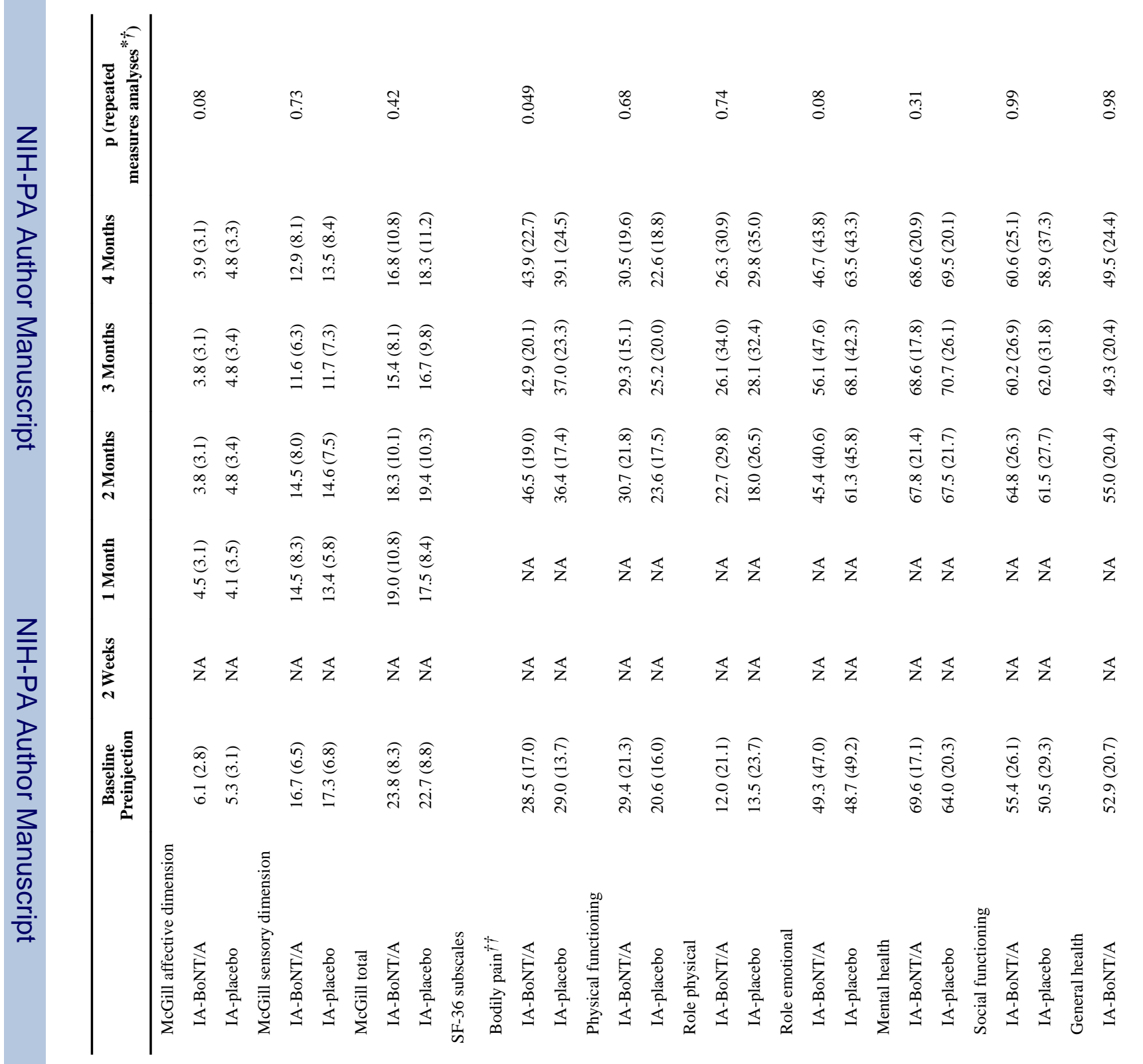




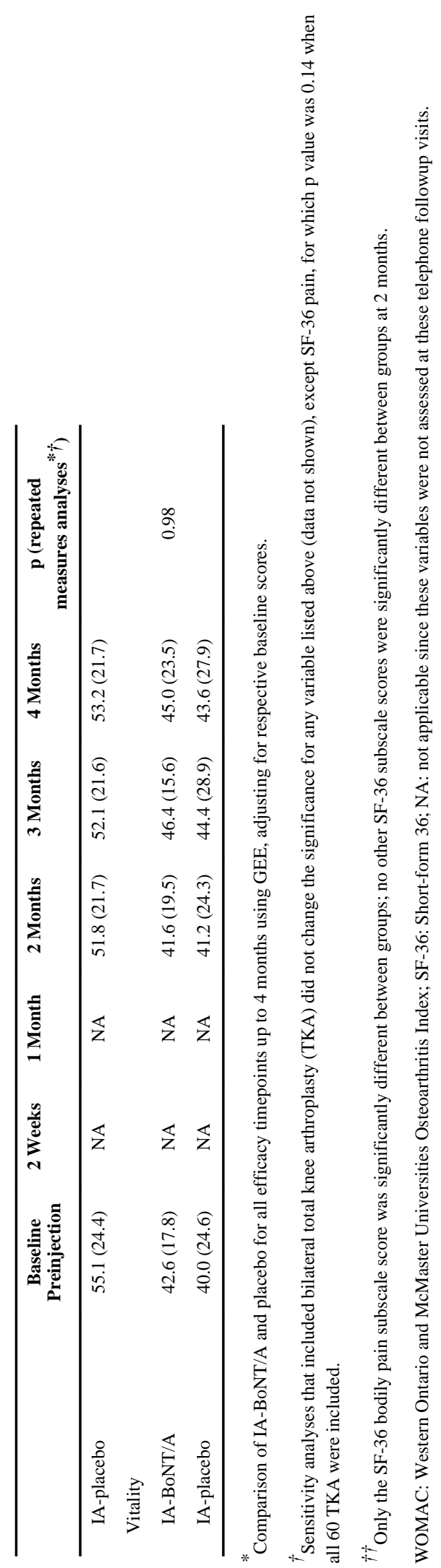

J Rheumatol. Author manuscript; available in PMC 2011 January 11. 
Table 5

Adverse events and serious adverse events.

\begin{tabular}{|c|c|c|c|}
\hline Adverse Events & IA-BoNT/A Group & IA-placebo Group & p, BoNT/A vs Placebo \\
\hline Total adverse events & 66 & 73 & 0.76 \\
\hline Serious adverse effects ${ }^{*}$ & 5 & 11 & 0.47 \\
\hline No. patients with $\geq 1$ serious adverse events & 3 & 9 & 0.16 \\
\hline \multicolumn{4}{|l|}{ Most common side effects } \\
\hline Dry mouth & 4 & 3 & \\
\hline Accidental injury & 11 & 7 & \\
\hline Back pain & 3 & 4 & \\
\hline Upper respiratory symptoms/infection & 10 & 10 & \\
\hline Difficulty swallowing & 0 & 4 & \\
\hline Headache & 3 & 2 & \\
\hline Nausea & 2 & 1 & \\
\hline Scheduled procedure & 3 & 5 & \\
\hline \multicolumn{4}{|l|}{ Local side effects } \\
\hline Pain in the study joint & 6 & 2 & \\
\hline Muscle weakness around the study joint or knee "giving out" & 4 & 2 & \\
\hline \multicolumn{4}{|l|}{ Serious adverse effects } \\
\hline \multicolumn{4}{|l|}{ Cardiac } \\
\hline Chest pain/new diagnosis of coronary artery disease & 0 & 3 & \\
\hline Supraventricular tachycardia & 0 & 1 & \\
\hline \multicolumn{4}{|l|}{ Pulmonary } \\
\hline Decreased saturation & 0 & 1 & \\
\hline Pneumonia & 0 & 1 & \\
\hline \multicolumn{4}{|l|}{ Other } \\
\hline Subarachnoid hemorrhage & 1 & 0 & \\
\hline Atypical chest pain & 1 & 0 & \\
\hline Seafood allergy & 1 & 0 & \\
\hline Depression & 2 & 0 & \\
\hline Gouty arthritis & 0 & 1 & \\
\hline Influenza & 0 & 1 & \\
\hline Cellulitis and septic arthritis & 0 & 1 & \\
\hline Deep vein thrombosis & 0 & 1 & \\
\hline Lethargy and decreased appetite & 0 & 1 & \\
\hline
\end{tabular}

